\title{
O PAPEL DAS CAPACIDADES DINÂMICAS NA INTERNACIONALIZAÇÃO DE EMPRESAS
}

\author{
Caroline Kretschmer e Ivan Lapuente Garrido ${ }^{1}$ \\ Universidade do Vale do Rio dos Sinos - Unisinos, Rio Grande do Sul, (Brasil)
}

\section{DETALHES DO ARTIGO}

\section{Histórico do Artigo:}

Recebido em: 23 de agosto de 2018

Aceito: 11 de fevereiro de 2019

Disponível online: 26 de março de 2019

Sistema de revisão "Double blind review"

\section{Editor Científico}

Ilan Avrichir

\section{Palavras-chaves:}

Capacidades Dinâmicas

Internacionalização

Estratégia

\begin{abstract}
RESUMO
O objetivo desta pesquisa é contribuir para a literatura de Capacidades Dinâmicas e Negócios Internacionais evidenciado quais os recursos organizacionais permitem a inserção e permanência em mercados externos, bem como as capacidades que são desenvolvidas por empresas que se internacionalizam e se mantêm no contexto internacional. Para tanto, buscou-se articular a lente teórica das capacidades dinâmicas e sua relação com a atuação em mercado internacionais, e aliar a literatura que aborda a internacionalização organizacional. Desenvolveu-se a pesquisa empírica por meio da análise do processo de internacionalização e do comportamento estratégico de duas empresas brasileiras. Realizou-se um estudo de caso múltiplo e qualitativo, os dados foram coletados por meio de entrevistas semiestruturadas e documentos, sendo analisados mediante a análise de conteúdo. Os resultados sustentam que a internacionalização se mostrou viável e alicerçada na articulação das capacidades dinâmicas que moldam uma arquitetura organizacional competitiva e na constante percepção dos gestores de novas oportunidades de atuação internacional provenientes de seus clientes. $\mathrm{O}$ artigo contribui para as duas vertentes teóricas propondo um framework para concatenalas, evidenciando os recursos, caminhos e capacidades necessárias para a perenidade e expansão em contextos internacionais, assim como a relevância das Capacidades Dinâmicas na internacionalização organizacional.
\end{abstract}

(C) 2018 Internext | ESPM. Todos os direitos reservados!

\section{INTRODUÇÃO}

O contexto macroeconômico mundial vem sofrendo grandes modificações ao longo dos anos, alterando continuamente a forma de atuação das organizações e impondo novos desafios e necessidades para a sobrevivência e sustentabilidade dos negócios. No ambiente empresarial atual, as contínuas mudanças nas demandas de clientes, nos panoramas competitivos e nas regulamentações governamentais são impostas às empresas (Zimmermann \& Birkinshaw, 2016). Assim, a vantagem competitiva dos negócios empresariais se encontra relacionada com o desenvolvimento e implantação de ativos intangíveis, com os relacionamentos e com o capital humano (Teece, 2014a).

Nesse aspecto, emerge a perspectiva das capacidades dinâmicas (CDs), abordagem que busca explicar o desempenho das empresas em economias globalizadas, baseadas em conhecimento e na qual dominam os ativos intangíveis (Teece, Pisano, \& Shuen, 1997). Trata-se de uma vertente de estudos que busca explicar como as empresas podem alcançar e sustentar uma vantagem competitiva (Peteraf, Di Stefano, \& Verona, 2013). Esta abordagem postula que, em regime de mudança rápida, o sucesso organizacional exige das empresas habilidades superiores em processos organizacionais, gerenciais e tecnológicos. Tais habilidades possibilitam a identificação e o aproveitamento de novas oportunidades, bem como a manutenção da competitividade por meio do incremento, proteção e reconfiguração dos ativos tangíveis e intangíveis da empresa (Teece et al., 1997; Teece, 2007). A orquestração dessas capacidades habilita as empresas a se tornarem e permanecerem ágeis em mercados domésticos e internacionais, como também evidenciam de que modo uma organização desenvolve pontos fortes, os sintoniza ao ambiente 
de negócios e os molda ao seu favor (Al-Aali \& Teece, 2014a).

A perspectiva das CDs se relaciona com os estudos relativos aos processos de internacionalização organizacional. As empresas que atuam em contextos internacionais, geralmente, estão expostas a ambientes altamente dinâmicos e caracterizados por rápidas mudanças, o que torna primordial o desenvolvimento de CDs para obtenção do desempenho internacional positivo (Augier \& Teece, 2007). Essas capacidades permitem operar de formar ágil tanto em âmbito doméstico quanto internacional, com flexibilidade estrutural e alta capacidade de aprendizagem e resposta (Teece, 2014a). Desse modo, as operações em mercados internacionais requerem CDs para habilitar a assimilação de conhecimentos e a incorporação desses aprendizados nas operações e na estratégia organizacional (Frasquet, Dawson, \& Mollá, 2013).

A expansão internacional se estrutura na base de recursos e ativos da empresa e nas idiossincrasias gerenciais, de modo que a internacionalização demonstra o acúmulo de conhecimento produtivo vinculado à trajetória organizacional (Knudsen \& Madsen, 2002). Nesse aspecto, tal processo é viabilizado por meio do desenvolvimento de vantagens específicas da empresa decorrentes da construção e articulação de CDs (Vahlne \& Johanson, 2017). Assim, "as capacidades dinâmicas estão no centro da competitividade global das empresas" e são desenvolvidas no âmbito doméstico e estendidas para o mercado internacional por organizações que se internacionalizam (Lessard, Teece, \& Leih, 2016a, p. 222). A internacionalização, neste cenário, é vislumbrada como uma decorrência da trajetória organizacional sustentada pelas CDs desenvolvidas nas operações domésticas ao longo do tempo. As empresas que atuam internacionalmente são laboratórios naturais para observar como as CDs atravessam as fronteiras contextuais, que podem ser uma indústria, uma orientação estratégica, países, ecossistemas ou blocos regionais (Lessard, Teece, \& Leih, 2016b). A compreensão do processo de desenvolvimento e articulação dessas capacidades nas operações em mercados externos amplia o entendimento da trajetória organizacional e a relevância das CDs para a perenidade desses negócios. A permanência e a evolução das empresas em mercados externos, ao longo do tempo, estão atreladas ao desenvolvimento de CDs no domínio das iniciativas internacionais (Lessard et al., 2016a).
Portanto, uma pesquisa central para aqueles que estudam CDs e Negócios Internacionais (NIs), que ainda se encontra em aberto, reside na investigação das circunstâncias em que os ativos intangíveis podem ser implantados, combinados e redistribuídos de forma lucrativa em outros contextos (Lessard et al., 2016b).

Conforme postulam Lessard et al. (2016b), mostra-se necessária a construção de estudos que considerem os contextos em que as CDs são desenvolvidas e exercidas e o aprofundamento da literatura de CDs e NIs por meio da abordagem de questões, como: "[...] quais capacidades podem permitir que uma empresa seja incorporada localmente em um contexto distintamente estrangeiro? [...] quais são os recursos que permitem que uma empresa local bem-sucedida amplie suas vantagens em outro lugar"? (p. 166). Por conseguinte, o presente estudo enfoca estas questões evidenciando os recursos e capacidades necessários para a internacionalização e buscando compreender o desenvolvimento e mobilização das CDs ao longo das trajetórias organizacionais no contexto doméstico e internacional. Diante disso, a pergunta que norteia esse estudo é apresentada como: qual o papel das capacidades dinâmicas na internacionalização e na manutenção da presença das empresas em mercados internacionais?

O presente trabalho aborda esse objetivo por meio da análise do processo de internacionalização e do desenvolvimento de CDs em empresas pertencentes à indústria de Tecnologia da Informação. Realizou-se um estudo de caso múltiplo, com duas empresas brasileiras, que atuam há mais de 20 anos no mercado; com ampla atuação em diversas regiões do Brasil; que exercem operações em mercados internacionais; e têm sua gestão inteiramente brasileira. A pesquisa desenvolvida analisou os movimentos estratégicos ocorridos ao longo da história dessas empresas, com foco nos eventos relacionados ao processo de internacionalização. A partir disso, avaliou-se a presença, construção e articulação de CDs nesses movimentos e qual relação essas capacidades exercem na trajetória das empresas. Assim, o estudo contribui para a ampliação dos estudos no escopo da gestão estratégica e da literatura de NIs, apresentando a concatenação das duas lentes teóricas e abordando a internacionalização por meio da ótica de recursos e capacidades organizacionais. Ademais, a pesquisa também contribui para 
O Papel das Capacidades Dinâmicas na Internacionalização de Empresas

evidenciar a articulação das CDs no âmbito doméstico e internacional e os recursos organizacionais que permite a inserção e permanência em mercados externos.

Para abordar o estudo proposto, na seção a seguir, apresenta-se a literatura sobre as estratégias de internacionalização, seguido pela perspectiva das CDs, seus elementos, sua relação com a internacionalização. A terceira seção descreve a abordagem empírica da pesquisa e a quarta seção contempla as análises e interpretações dos casos estudados, bem como o framework proposto. Por fim, constam as considerações finais, direções para futuras pesquisas e as referências consultadas.

\section{INTERNACIONALIZAÇÃO ORGANIZACIONAL}

$A$ atuação em mercados internacionais se configura como um processo multifacetado e desenvolvido ao longo do tempo, sendo um tema amplamente estudado na literatura desde 1970 (Welch \& Paavilainen-Mäntymäki, 2014). As correntes de estudo na área da internacionalização são formadas por diferentes linhas de pesquisa sendo que, dois enfoques diferenciados obtiveram destaque nas pesquisas: a abordagem econômica, que se baseia em decisões racionais e visa maximizar lucros; e a abordagem comportamental, que foca nas percepções, atitudes, e restrições cognitivas das decisões gerenciais (Andersen \& Buvik, 2002). O presente estudo se foca na linha comportamental e, portanto, aprofunda nesta temática.

As teorias comportamentais abarcam o comportamento estratégico organizacional. A Escola Nórdica de Internacionalização da Firma, foi pioneira nesta abordagem na análise do processo de atuação em mercados externos (Björkman \& Forsgren, 2000). O modelo do processo de internacionalização nesta linha postula a ideia de que a aprendizagem experimental é o fator chave para as mudanças e no delineamento da trajetória internacional da empresa, bem como a aprendizagem e experiência prévia sustentam as escolhas de localização para atuar nos mercados externos (Kim \& Aguilera, 2015).

Esses estudos evoluíram e passaram a abranger as críticas e as mudanças nos ambientes econômicos e de regulação, bem como em diversos aspectos do comportamento das empresas (Johanson \& Vahlne, 2009; Vahlne \& Johanson, 2013). Um tópico relevante neste âmbito trata da importância das redes de relacionamento internas e externas firmadas pelas empresas que atuam internacionalmente (networks). Nessa abordagem, os mercados são retratados como redes de relações entre empresas e para que os negócios ocorram, estas organizações necessitam desenvolver um conhecimento aprofundado uma sobre as outras (Johanson \& Mattson, 1988; Vahlne \& Johanson, 2013). As networks criadas entre diferentes agentes (clientes, fornecedores, órgãos oficiais, parceiros de alianças, entre outros) se apresentam como uma forma eficaz da manutenção do mercado externo (Welch \& Welch, 1996).

As networks influenciam aspectos de empreendedorismo internacional, sendo outra relevante linha de pesquisa no escopo da abordagem comportamental de internacionalização (Coviello \& Munro, 1995). Os relacionamentos construídos e as ligações desenvolvidas pelas empresas em mercados externos permitem o acesso a novas experiências, recursos e conhecimentos que impulsionam a sua capacidade para penetrarem em mercados internacionais (Pla-Barber \& Escribá-Esteve, 2006). Esse processo exige uma gestão dinâmica e proativa, a qual se encontra em uma orientação empreendedora (Pla-Barber \& Escribá-Esteve, 2006).

A orientação empreendedora se associa à capacidade de inovação, à visão gerencial e à postura competitiva no exterior, refletindo a proatividade global na busca de mercados internacionais (Knight, 2001). Essa orientação retrata um comportamento organizacional relacionado à busca de oportunidades, tomada de riscos e inovação, sendo, em geral, positivamente associado ao desempenho e competitividade das empresas (Thanos, Dimitratos, \& Sapouna, 2017). As empresas empreendedoras são mais propensas a assumir riscos, a buscar novas tecnologias, a desempenhar funções organizacionais de maneira diferenciada e a lançar empreendimentos internacionais mais ousados. Assim, propiciam o acesso a novas oportunidades e negócios, expandindo sua base de conhecimentos, seus recursos e suas capacidades (Knight, 2001).

A orientação empreendedora e a participação da empresa em uma rede na qual ela interage com atores que operam nacional ou internacionalmente, auxiliam a inserção da empresa no mercado internacional. Além, disso, habilitam a escolha de uma estratégia de internacionalização que pode ser resultado da busca de mercados ou a de seguir o cliente (Majkgard \& Sharma, 1998). A estratégia de seguir o cliente tem uma estreita 
ligação com as networks e assume que a empresa opera em uma rede na qual os seus parceiros atuam internacionalmente e pressionam os demais membros a também atuarem. Nos primeiros anos de inserção internacional, suas operações nos mercados externos se baseiam nos recursos fornecidos pela rede doméstica (Majkgard \& Sharma, 1998).

A escolha por essa estratégia é predominantemente efetuada por empresas do setor de serviços, que tradicionalmente iniciam sua atuação internacional quando seus clientes se internacionalizam (Grönroos, 1999). As características das empresas de serviços, como intangibilidade, heterogeneidade, inseparabilidade e a estreita relação entre produtor e consumidor as tornam propicias a assumir formas de internacionalização diferente de indústrias de transformação (Lommelen \& Matthyssens, 2005). Além disso, essas empresas, em geral, necessitam seguir seus clientes no exterior a fim de evitar que concorrentes estrangeiros passem a atendê-los e, eventualmente, possam entrar também no mercado doméstico e prejudicar sua futura participação de mercado e lucratividade (Boehe, 2016). Sendo assim, a internacionalização dessas empresas reflete, principalmente, as oportunidades decorrentes dos contatos externos, intercâmbio social e a confiança estabelecida com os clientes e colaboradores.Esta estratégia busca capitalizar as relações empresariais preestabelecidas (Lommelen \& Matthyssens, 2005). A empresa fornece serviços similares ao mercado interno para os seus clientes no exterior, de forma a nutrir e reforçar o relacionamento existente (Blomstermo et al., 2006). A empresa prestadora de serviços obtém uma vantagem, na medida em que começa sua operação internacional com clientes que possui laços fortes (Huijs, Jansen, \& Brinkkemper, 2015). Ademais, o conhecimento gerado e as informações coletadas sobre as atividades internacionais de seus clientes podem ser transferidos para âmbito doméstico, permitindo um melhor atendimento nas operações nacionais dos clientes (Boehe, 2016).
A internacionalização é compreendida assim, como uma atividade de aprendizado e que envolve os recursos e capacidades internas da empresa (Majkgard \& Sharma, 1998). Os conhecimentos necessários nesse processo podem ser potencializados por meio de suas relações atuais de intercâmbio e o conhecimento experimental gerado exerce uma relevante influência na internacionalização da empresa (Eriksson, Johanson, \& Majkgard, 1997). Apesar dessa forte ligação com clientes e o papel dos relacionamentos na internacionalização das empresas de serviços, esse processo também requer movimentos específicos e internos da empresa em aprender, organizar e gerenciar os esforços para essa estratégia (Eriksson et al., 1997).

A perspectiva da estratégia de seguir o cliente, das networks e do empreendedorismo internacional configuram-se como expoentes no embasamento da competitividade das empresas que atuam no mercado externo. A postura agressiva e proativa da empresa, aliada às redes de relacionamento constituídas com diferentes agentes, permite acessar e desenvolver capacidades e recursos diferenciados e de difícil replicação por parte dos concorrentes (Johanson \& Vahlne, 2009; Vahne \& Johanson, 2017). Essas vertentes de pesquisa se vinculam à perspectiva das CDs, onde a capacidade da empresa em desenvolver e articular ativamente seus recursos tangíveis e intangíveis para responder às mudanças ambientais aliadas a uma estratégia adequada é a base para se obter um desempenho superior (Teece, 2014b). Nesse estudo, a internacionalização é entendida a partir da vertente das networks e sua influência na adoção de estratégia de internacionalização de seguir o cliente, conduzindo ao desenvolvimento de capacidades, recursos e experiências necessários para delinear o processo de inserção internacional por este caminho. A Figura 1 demonstra 0 encadeamento dessas perspectivas teóricas e se vincula à pesquisa realizada, em que a lente teórica das CDs se atrela às estratégias de internacionalização.

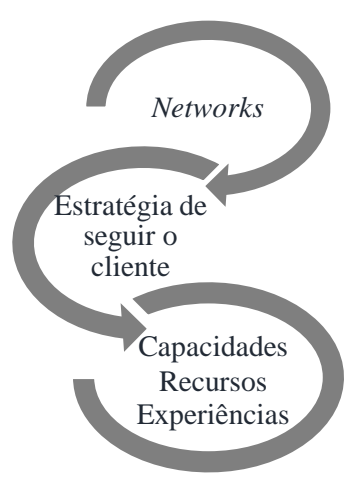

Figura 1. A internacionalização na perspectiva do estudo Fonte: Elaborado pelos autores 
Na formulação e desempenho da estratégia em ambiente global, fatores como visão empreendedora, experiência internacional dos gestores e o estado de alerta para as oportunidades também devem ser considerados e refletem uma posição ativa em relação aos desafios externos (Crick, 2009). O êxito na internacionalização das empresas passa a se estabelecer a partir da sua carteira de recursos e capacidades e, principalmente, na sua capacidade de se ajustá-los e reconfigurá-los de acordo com as mudanças internacionais. Nesse contexto, o processo de internacionalização passa a exigir uma análise por meio da ótica de recursos e capacidades da empresa (Prange \& Verdier, 2011; Vahne \& Johanson, 2017). Assim, para abarcar e delinear de forma mais aprofundada o vínculo entre o processo e as teorias de internacionalização de empresas e a perspectiva das CDs, apresenta-se na próxima seção os conceitos e elementos fundamentais que relacionam essas duas abordagens.

\section{CAPACIDADES DINÂMICAS E A INTERNACIONALIZAÇÃO}

A perspectiva das CDs situa-se dentro da vertente de estudos da administração estratégica e tem como foco a estruturação e análise das possibilidades de construção e manutenção da competitividade organizacional em ambientes mutáveis e complexos (Helfat et al., 2009; Teece, 2014a). Há uma variedade de interpretações e concepções acerca do tema, os conceitos e os elementos basilares são mutáveis dentre os diferentes pesquisadores (Di Stefano; Peteraf, \& Verona, 2010). Entretanto, as definições de CDs convergem no sentido de identificá-las como processos organizacionais que permitem mudar a base recursos da empresa (Helfat et al., 2009). O conceito seminal desta abordagem apresenta as CDs como "[...] a habilidade da empresa de integrar, construir e reconfigurar competências internas e externas para abordar rapidamente mudanças no ambiente" (Teece et al., 1997, p. 516).

A fundamentação das CDs reside em elementos estratégicos contemplados em três categorias: processos, posições e caminhos (Teece et al., 1997). Os processos se traduzem na maneira de desempenhar as atividades e rotinas; as posições evidenciam as tecnologias, a propriedade intelectual, a base de clientes e relação com fornecedores e agentes que a empresa possui; e os caminhos apresentam a dependência da trajetória organizacional, os retornos de seus negócios e quais alternativas estratégias estão disponíveis. Nesse sentido, os processos moldam as posições atuais da empresa e seus caminhos evolutivos, de modo a delinear o cerne das CDs organizacionais.

As CDs podem ser desagregadas dentro de três grandes pilares que abarcam suas diversas classes (Teece, 2007). O primeiro deles é a capacidade de sentir e moldar oportunidades e ameaças (sense), em que se desenvolvem atividades como a criação; aprendizagem; pesquisa; sondagem das possibilidades tecnológicas, necessidades dos clientes e da evolução estrutural de mercados e concorrentes. O segundo é a apreensão dessas oportunidades (seize), por meio do desenvolvimento de novos processos, produtos e serviços. Por fim, tem-se a reconfiguração dos ativos tangíveis e intangíveis do negócio da empresa (reconfiguring ou transforming), que permite a avaliação das atividades organizacionais e a reformulação de rotinas, realinhamento de atividades e adaptação das unidades de negócios.

$\mathrm{Na}$ internacionalização organizacional, a construção e articulação das CDs são de extrema relevância e apresentam algumas especificidades. Neste contexto, a competitividade e mutabilidade dos ambientes são maiores, assim como a exposição à concorrência global, as diferentes fontes de inovação e a dispersão da produção (Teece, 2014a). No processo de internacionalização, tem-se a necessidade de uma orientação empreendedora para gerenciar os empreendimentos internacionais, o conhecimento e a aprendizagem organizacional acerca dos países a fim de lidar com o incremento da complexidade e do dinamismo do ambiente internacional (Frasquet et al., 2013; Vahne \& Johanson, 2017). Assim, a estrutura das CDs fornece os subsídios para auxiliar as organizações nesse processo de atuação internacional.

As CDs, articuladas nas operações em mercados externos, abarcam o reconhecimento das características específicas dos países em que atua e a adaptação, integração e reconfiguração de ativos internos e externos para aproveitar oportunidades no mercado global (Griffith \& Harvey, 2001). A gestão das atividades internacionais requer elementos como flexibilidade, agilidade, empreendedorismo, escolhas inteligentes de investimentos e aprendizado contínuo, que são centrais na perspectiva das CDs 
(Pitelis \& Teece, 2010). Portanto, as competências gerenciais, em conjunto com as CDs, sustentam o gerenciamento das operações internacionais.

A internacionalização requer CDs para compensar as desvantagens da estranheza do país hospedeiro e manter a competitividade perante os rivais locais e concorrentes estrangeiros. Por conseguinte, esse movimento fornece novas oportunidades para extrair retornos a partir dos recursos atuais da empresa e construir novas capacidades (Luo, 2001). Dessa forma, a compreensão do processo de mudanças e reconfiguração dos recursos organizacionais para o desenvolvimento organizacional se mostra fundamental para compreender as fontes de vantagem competitiva que viabilizam a internacionalização bem-sucedida (Vahne \& Johanson, 2017).

Assim, os três pilares das CDs, conforme proposto por Teece (2007) podem ser estendidos para as operações internacionais. $\mathrm{Na}$ identificação de oportunidades, faz-se necessário incluir o reconhecimento da demanda latente da escala global; na apreensão de oportunidades inclui-se coordenação, controle e influência dessas oportunidades e dos demais ativos para estabelecer diferentes formas de atuação em perspectivas internacionais; e por fim, na dimensão da reconfiguração, tem-se alterações de modelos de negócios, cultura organizacional, reformulação de linhas de produtos e serviços e renovação de instalações em nível interno e mundial (A-Alli \& Teece, 2014).

Alguns trabalhos empíricos analisaram a construção e articulação das CDs no contexto da internacionalização organizacional. Diferentes estudos vinculam as CDs com a gestão e incremento do conhecimento e da aprendizagem organizacional para as empresas atuarem em mercados internacionais (Fan, Cui, Li, \& Zhu, 2016; Pehrsson et al., 2015; Villar, Alegre, \& Pla-Barber, 2014). Outros assumem as CDs como a capacidade de estabelecer parcerias no mercado internacional (Chang, Chen, \& Huang, 2015) ou como a capacidade exploratória para criar valor ao nível da empresa no processo de internacionalização (Lew, Sinkovics, \& Kuivalainen, 2013). Diversas pesquisas utilizaram a lente teórica das CDs para analisar o desenvolvimento e o desempenho de novos empreendimentos internacionais e Born Globals (Evers; Andersson, \& Haniibal, 2012; Jantunen et al., 2005; Khalid \&
Larimo, 2012; Khavul, Peterson, \& Mullens, 2010; Monferrer; Blesa; Ripollés, 2015; Turcan \& Juho, 2016; Weerawardena et al., 2007; 2015), bem como de franquias internacionais (El Akremi; Perrigot \& Piot-Lepetit, 2015); fábricas globais (Eriksson, Nummela, \& Saarenketo, 2014); multinacionais (Chakrabarty \& Wang, 2012; Da Costa \& Porto, 2014; Floriani; Borini, \& Fleury, 2009; Parente, Baack, \& Hahn, 2011; Vahlne \& Ivarsson, 2014; Vahlne \& Jonsson, 2017; Werlang, Dalbosco, Floriani, \& Rosetto, 2014), pequenas e médias empresas (Pinho \& Prange, 2016; Swoboda \& Olejnik, 2016; Tallott \& Hilliard, 2016) empresas exportadoras (Moreira \& Forte, 2006; Rodriguez; Wise, \& Martinez, 2013). Outros estudos evidenciaram que as empresas se envolvem em joint ventures internacionais para desenvolver CDs necessárias para atuarem internacionalmente (Fang \& Zou, 2009) ou desenvolvem CDs para atuarem na rede educacional global, no contexto de serviços de ensino superior (Butler \& Soonties, 2015).

O panorama geral desses estudos apresenta a articulação das CDs diretamente relacionadas ao início da inserção internacional, porém, não avaliam o papel destas capacidades na permanência das empresas nos mercados internacionais. Além disso, não evidenciam a relevância da construção das CDs ao longo da trajetória da empresa no mercado doméstico para habilitar a adoção de uma estratégia de expansão internacional. Assim, o presente estudo aborda tais questões evidenciando no âmbito empírico a relação entre as CDs e a internacionalização, ao longo das trajetórias organizacionais.

\section{MÉTODO}

O estudo realizado se utilizou de uma abordagem qualitativa, descritiva e exploratória. Realizou-se um estudo de caso múltiplo em duas empresas brasileiras do setor de TI. A escolha dos casos partiu do mapeamento do setor, tendo como critérios para a seleção dos participantes serem empresas pertencentes à Indústria Brasileira de Software e Serviços; de origem nacional; terem passado por mudanças estratégicas significantes (modificações em modelos de negócios; realinhamento de recursos e ativos e inserção em mercados internacionais); consolidadas nacionalmente e com atuação internacional.

O primeiro caso é o Grupo Alfa, caracterizado como uma plataforma de informação, oferecendo 
serviços de consultoria em TI, serviços SAP, outsourcing, fábrica de software e serviços de gerenciamento de aplicações, fundada em 1990 e atendendo médias e grandes empresas. Com seis unidades no Brasil, uma Unidade de Pesquisa e Desenvolvimento no RS e um escritório nos EUA. O segundo caso é o Grupo Beta, formado por empresas que implantam e integram serviços e soluções em TI no mercado latino-americano, tendo bases de atendimento em São Paulo, Rio de Janeiro, Curitiba, Londrina, Belo Horizonte e em Porto Alegre, com escritórios comerciais no Chile, na Argentina e nos Estados Unidos.

A coleta de dados foi realizada por meio de quinze entrevistas semiestruturadas com doze entrevistados, sendo oito no Grupo Alfa e sete no Grupo Beta, no ano de 2016. Os entrevistados foram sócios e gestores do alto escalão (diretores e gerentes de área) pertencentes a diferentes setores, que fizeram parte da história das organizações ou tinham amplo conhecimento do caminho percorrido por elas.

A identificação das empresas e dos entrevistados foram preservadas. As entrevistadas foram gravadas e transcritas para compor as análises. As transcrições geraram 233 páginas, sendo 130 páginas dos entrevistados da empresa Alfa e 103 páginas dos entrevistados da empresa Beta. O tempo total das entrevistas foi de 14 horas, sendo 7 horas e 50 minutos na empresa Alfa e 6 horas e 10 minutos na empresa Beta. Além dos dados das entrevistas também foram utilizados documentos internos disponibilizados pelas empresas e documentos externos para complementar a pesquisa realizada. Os documentos internos foram constituídos por livros e relatórios institucionais que relatam detalhadamente as histórias organizacionais e seus movimentos estratégicos. Os documentos externos das empresas estudadas compilados para análise contemplaram reportagens veiculadas em jornais de âmbito nacional e regional; revistas (por exemplo, Destaque Gaúcho e 100 Marcas do Rio Grande do Sul); livro (Knebel, 2010); e publicações em mídias digitais. Os materiais divulgavam as operações, atividades, mudanças estratégicas e premiações recebidas pelos casos analisados. A concatenação dos documentos internos e externos com as entrevistas realizadas permitiram convergir as informações acerca das peculiaridades das empresas, das suas conjunturas internas, dos seus ambientes de negócio e das trajetórias organizacionais.

A apuração dos resultados foi realizada por meio de análise de conteúdo e foram categorizados da seguinte forma: (1) movimentos estratégicos relacionados com o processo de internacionalização ocorridos nas empresas estudadas; e (2) as CDs desenvolvidas, decompostas em seus três pilares (detecção, apreensão e transformação), de acordo com a ótica de Teece (2007). A Figura 2 apresenta as categorias de análise que sustentaram a pesquisa realizada e o detalhamento de sua composição. Realizou-se um mapeamento para compreender a história de cada empresa, identificando as mudanças estratégicas ocorridas e, principalmente, aquelas relacionadas com o processo de internacionalização. A partir disso, analisou-se o desenvolvimento e a articulação das CDs nesses momentos de mudança pertinentes à inserção internacional.

\begin{tabular}{|c|c|c|c|}
\hline Categorias & Sub categoria & Aspectos observáveis & Referências \\
\hline $\begin{array}{l}\text { Movimentos } \\
\text { estratégicos }\end{array}$ & Internacionalização & $\begin{array}{l}\text { Abertura de filiais; abertura de escritórios } \\
\text { comerciais; projetos e atividades desenvolvidas } \\
\text { em âmbito internacional. }\end{array}$ & - \\
\hline \multirow{2}{*}{$\begin{array}{l}\text { Capacidades } \\
\text { Dinâmicas }\end{array}$} & $\begin{array}{l}\text { Detectar e moldar } \\
\text { oportunidades }\end{array}$ & $\begin{array}{l}\text { Investimentos em P\&D; geração de base externa } \\
\text { de pesquisas; aquisição de conhecimentos; } \\
\text { desenvolvimento de rotinas de criação de } \\
\text { conhecimentos; monitoramento das atividades } \\
\text { de concorrentes, clientes e fornecedores; } \\
\text { desenvolvimento de processos para identificar } \\
\text { mercados-alvo. }\end{array}$ & \multirow{2}{*}{$\begin{array}{l}\text { A-Alli \& Teece }(2014) ; \\
\text { Teece }(2007) ; \text { Teece } \\
(2014 a, 2014 b)\end{array}$} \\
\hline & $\begin{array}{l}\text { Apreender as } \\
\text { oportunidades }\end{array}$ & $\begin{array}{l}\text { Desenvolvimento de produtos, processos e } \\
\text { serviços; criação, ajuste e aprimoramento dos } \\
\text { modelos de negócio; aquisição de novas } \\
\text { tecnologias; decisões estratégicas; } \\
\text { transformação de rotinas; assimilação de } \\
\text { conhecimentos. }\end{array}$ & \\
\hline
\end{tabular}




\begin{tabular}{|c|c|c|c|}
\hline & $\begin{array}{c}\text { Gerenciar ameaças e } \\
\text { transformar }\end{array}$ & $\begin{array}{l}\text { Descentralização e gestão colaborativa; } \\
\text { governança; coespecialização; flexibilidade e } \\
\text { capacidade de resposta; gestão do conhecimento } \\
\text { (exploração, compartilhamento, codificação); } \\
\text { redes de colaboração; abandono de recursos. }\end{array}$ & \\
\hline $\begin{array}{c}\text { Internacionaliza } \\
\text { ção }\end{array}$ & $\begin{array}{c}\text { Estratégias e } \\
\text { processos }\end{array}$ & $\begin{array}{l}\text { Motivos para se internacionalizar e modos de } \\
\text { entrada; níveis de controle e riscos assumidos; } \\
\text { adaptações para as atividades relacionadas às } \\
\text { operações em mercados externos; dificuldades e } \\
\text { exitos; benefícios. }\end{array}$ & $\begin{array}{l}\text { Boehe (2016); Eriksson, } \\
\text { Johanson, \& Majkgard } \\
\text { (1997); Johanson \& } \\
\text { Vahlne (2009); Knight } \\
\text { (2001); Majkgard \& } \\
\text { Sharma (1998). }\end{array}$ \\
\hline
\end{tabular}

Figura 2. Categorias de análise

Fonte: Elaborado pelos autores

\section{RESULTADOS}

Nesta seção, apresenta-se o panorama de atuação internacional de cada um dos casos, a análise geral dos achados e se propõe um framework que integra as perspectivas analisadas no estudo. A Figura 3 exibe os eventos relacionados com os movimentos internacionais realizados pelas empresas estudadas e que foram analisados a partir da ótica das CDs.

\begin{tabular}{|l|l|}
\hline \multicolumn{1}{|c|}{ Empresa Alfa } & \multicolumn{1}{c|}{ Empresa Beta } \\
\hline \multirow{2}{*}{$\begin{array}{l}\text { - Projetos internacionais em Portugal, no Peru, Colômbia } \\
\text { e México }\end{array}$} & - Projeto internacional no Uruguai \\
& - Filiais no Chile e Colômbia \\
- Escritórios comerciais nos Estados Unidos, Espanha e & Argentina \\
\hline
\end{tabular}

Figura 3. Movimentos internacionais realizados pelas empresas

Fonte: Elaborado pelos autores

Na empresa Alfa, o processo de internacionalização se configurou como uma evolução dos seus negócios e se iniciou a partir da base dos atendidos no mercado doméstico. As iniciativas de atuação internacional desenvolvidas, desde seu primeiro movimento em 2001, sempre foram realizadas por meio de seus clientes. Alguns elementos possibilitaram a construção de um posicionamento de mercado e um reconhecimento, por parte dos clientes, de que a Alfa apresentava os atributos necessários para acompanhar os seus NIs. Destacam-se neste quesito, a sua trajetória ao longo do tempo, sua consolidação no mercado doméstico e o desenvolvimento e manutenção de CDs. Os entrevistados relataram que a reputação da empresa no Brasil é muito forte, principalmente pelas multinacionais estrangeiras que operam no país e que são atendidas pela Alfa. Com isso, essas multinacionais à conduzem para outros lugares do mundo para estender seus serviços prestados em contratos globais.

As características da empresa e sua capacidade de ser flexível e desenvolver um trabalho de nível elevado, permitem gerenciar os projetos internacionais e também ser percebida pelos clientes como um parceiro promissor para o desenvolvimento de seus negócios. No primeiro projeto internacional, realizado em Portugal, na Caixa Geral de Depósitos, o cliente conheceu o modelo de fábrica de software que a Alfa desenvolve no Brasil e solicitou um projeto para implementar a mesma configuração internacionalmente. Verifica-se que as capacidades da empresa, quando alinhadas com as necessidades dos mercados externos, facilitam a sua expansão internacional (Teece, 2014a). No processo vivenciado pela empresa Alfa, os projetos internacionais se tornaram mais facilitados em sua execução, pois os recursos estratégicos e as CDs gerenciadas pela empresa foram estendidas para esses novos mercados, proporcionando também a diminuição da estranheza com esses locais. Outro elemento desse panorama é o modo como a empresa iniciou sua internacionalização, que reflete uma característica do tipo de negócio. Empresas de serviços, tradicionalmente, iniciam sua ida para o exterior para seguir os clientes as quais elas estão oferecendo serviços em mercados domésticos (Blomstermo et al., 2006; Grönroos, 1999). Esse aspecto foi relatado nas entrevistas como uma característica do processo de internacionalização da empresa, o qual sempre se configura por meio dos seus clientes e das 
oportunidades que surgem a partir desses relacionamentos. A Alfa vai se expandindo e crescendo junto com eles, identificando novas oportunidades e conhecendo novos mercados por meio desse relacionamento.

Essa estratégia de internacionalização almeja construir relações de valor com seus clientes já estabelecidos, buscando fornecer mais ou menos os mesmos serviços aos clientes no exterior e, assim, crescer e fortalecer o relacionamento com eles (Blomstermo et al., 2006). Ademais, para as empresas que atuam em mercados internacionais é necessário que suas CDs sejam ampliadas e alavancadas frente àquelas empresas com foco exclusivamente interno (Teece, 2014a). A Figura 4 apresenta a análise das CDs desenvolvidas no processo de internacionalização da Alfa a partir dos três pilares das CDs proposto por Teece (2007).

\begin{tabular}{|l|l|l|}
\hline Identificação da Oportunidade & Apreensão da Oportunidade & \multicolumn{1}{|c|}{ Reconfiguração } \\
\hline $\begin{array}{l}\text { - A partir das necessidades dos } \\
\text { clientes e de sua expansão } \\
\text { internacional. }\end{array}$ & $\begin{array}{l}\text { - Treinamentos; } \\
\text { - Articulação de conhecimentos; } \\
\text { - Tomada de decisões estratégicas. }\end{array}$ & $\begin{array}{l}\text { Contínuo acompanhamento das } \\
\text { necessidades dos clientes em mercados } \\
\text { internacionais; } \\
\text { - Integração dos conhecimentos e de ativos } \\
\text { presentes no mercado doméstico para } \\
\text { abordar as exigências do ambiente } \\
\text { internacional. }\end{array}$ \\
\hline
\end{tabular}

Figura 4. Articulação das CDs na internacionalização da empresa Alfa

Fonte: Elaborado pelos autores

O processo de internacionalização da empresa Alfa deve ser analisado como uma consequência da trajetória organizacional. Também, faz-se necessário considerar o desenvolvimento de boas estratégias e o posicionamento da empresa perante o mercado, que a faz ser reconhecida como uma empresa de excelência nas suas linhas de negócio. Os dados coletados permitiram averiguar a presença de CDs dentro da empresa, como sua orientação para o mercado; o estabelecimento de relações de confiança e parceria com clientes, que permitem identificar, mapear e avaliar novas oportunidades e a evolução dos mercados em diferentes contextos de atuação. Além disso, a capacidade de reconfigurar suas atividades; de desenvolver tecnologias e mão de obra; de integrar e articular conhecimento, ativos e recursos entre o âmbito doméstico e internacional se evidenciam como CDs essenciais na atuação internacional.

Assim, tais elementos elevam continuamente o nível de competitividade da empresa Alfa, sendo primordiais para a permanência da empresa na gestão de projetos globais.

$\mathrm{Na}$ empresa Beta, o início do processo de internacionalização também se desenvolveu em virtude da necessidade de acompanhar a expansão dos clientes atendidos no Brasil. Além disso, a pressão de concorrentes, não só a nível nacional, influenciou a adoção dessa estratégia. A partir de
2005, os clientes passaram a solicitar que a Beta se tornasse um player global, a fim de atendê-los em contratos globais e, se a Beta não realizasse essa expansão internacional, correria o risco de perder grandes contratos. Assim, o crescimento das empresas brasileiras e seu desenvolvimento a nível internacional surgiram como uma oportunidade para a Beta iniciar o primeiro movimento estratégico de internacionalização. Neste cenário, a empresa abriu em 2005 a sua primeira filial, instalada no Chile e, posteriormente, na Colômbia, Estados Unidos, Espanha e Argentina. A abertura de escritórios comerciais e prestação de serviços em diversos contextos externos permitiu à Beta se defender de ameaças globais de grandes multinacionais de mercado. Essa conjuntura é corroborada pela literatura (Boehe, 2016), a qual argumenta que as empresas de serviços seguem seus clientes no exterior para evitar que concorrentes estrangeiros possam ampliar sua atuação e ingressar também no contexto doméstico, passando a atender seus clientes e prejudicando sua participação de mercado. Com isso, a Beta garantiu sua continuidade nos clientes, além de aumentar o volume de contratos. Nesse contexto, a questão de a empresa possuir uma estrutura adequada e ser competitiva nacionalmente, com a associação da sua imagem à Microsoft, permitiu ter uma maior aceitação em âmbito internacional. 
Apesar da internacionalização ser predominantemente para acompanhar os clientes, a unidade aberta no Chile foi um movimento exclusivo da empresa e não associado a clientes. Esse mercado foi escolhido pelo sócio fundador em virtude de sua proximidade com a cultura do país, com a lógica de trabalho desenvolvida e pelo seu conhecimento a respeito do funcionamento daquele mercado. Atualmente, 95\% da receita dessa unidade é local, ou seja, não são derivados de contratos globais. Para atender esse mercado, a empresa montou uma estrutura composta por profissionais chilenos para trabalhar na área comercial, pois a estrutura de custos, formas de trabalho, a cobrança de impostos, a cultura das empresas e a maneira de posicionamento da concorrência são muito distintas em comparação ao Brasil. No entanto, a empresa também enviou profissionais brasileiros que tem a cultura da Beta e um maior tempo de empresa para atuar junto aos colaboradores locais, para que se tornassem replicadores locais do modelo de trabalho da empresa.

A estratégia de internacionalização para seguir os clientes em mercados estrangeiros é adotada, predominantemente, por empresas do setor de serviços com a finalidade de capitalizar as relações já estabelecidas (Lommelen \& Matthyssens, 2005). Após o estabelecimento da presença no mercado externo, a motivação parece se alterar para o atendimento de clientes estrangeiros locais, e se adota uma abordagem de mercado. Essa estratégia conduz a empresa a fazer parte de uma rede internacional de câmbio, que auxilia na acumulação de conhecimentos experimental e nos procedimentos de tomada de decisões (Majkgard \& Sharma, 1998). No panorama vivenciando pela Beta, essa abordagem, assim como o Modelo de Uppsala são verificados na medida em que a empresa vem construindo sua internacionalização por meio de relacionamento e aprendizagem experimental para explorar e aproveitar as oportunidades.

O processo de expansão internacional após o período inicial e na busca de ampliar a atuação para além da base de clientes já atendida, também demandou algumas estratégias por parte da empresa para conquistar novos clientes e se tornar reconhecida como no Brasil. Os entrevistados relataram que primeiro é efetuado um mapeamento dos clientes da região, por meio de anuários e pesquisas na internet em conjunto com a definição do mercado que se deseja trabalhar: perfil do cliente; tamanho de faturamento; quantidade de funcionários; mercado público ou privado. Além disso, realizam-se buscas para verificar em qual região esses clientes-alvo estão distribuídos. Como estratégia, a empresa inicia o trabalho em clientes de segmentos em que ela já tenha casos de êxito, como negócios similares, de modo a gerar a percepção de ganhos e resultados para aquele potencial cliente.

Os benefícios desse trabalho desenvolvido também trouxeram impactos positivos para a atuação da empresa em âmbito nacional. O conhecimento adquirido por meio dessa expansão possibilitou a ampliação de atendimento para empresas internacionais instaladas no Brasil. Uma parcela significativa das grandes empresas brasileiras é gerida por estrangeiros, provenientes de outras culturas e que têm dificuldades de lidar com a conjuntura brasileira. Assim, a Beta, já tendo vivenciado diversas culturas e conhecendo a forma como essas pessoas pensam e suas lógicas de trabalho, pôde servir como um prestador de serviço para conectar esses gestores com a realidade brasileira.

Observa-se que o processo de internacionalização da Beta apresenta características semelhantes àquelas percebidas na empresa Alfa. A motivação para ingressar em mercados internacionais se deu por meio da necessidade e solicitação dos seus clientes.

Os clientes percebem a Beta como um parceiro preparado para auxiliar na sustentação dos seus negócios em âmbito nacional e internacional. Essa percepção é proveniente da trajetória trilhada pela empresa, principalmente na formatação de novos modelos de negócio, na busca de estar sempre à frente do mercado, de se expandir geograficamente e na constante reestruturação interna para se adaptar a novos contextos tecnológicos. As CDs da Beta de gerenciar, disseminar integrar conhecimentos entre o mercado doméstico e internacional; de coordenar uma base global de recursos distintos e de reconfigurar seus ativos, propiciam um posicionamento competitivo de difícil imitação pelos concorrentes.

A internacionalização da empresa reflete a capacidade da empresa de sustentar uma arquitetura organizacional distintiva, que contempla os três pilares das CDs, conforme consta na Figura 5. Isso habilita não somente acompanhar os clientes para mercados externos, como também permanecer nesses locais e ampliar seu escopo de atuação, explorando novos mercados. 


\begin{tabular}{|l|l|l|}
\hline \multicolumn{1}{|c|}{ Identificação da Oportunidade } & \multicolumn{1}{|c|}{ Apreensão da Oportunidade } & \multicolumn{1}{c|}{ Reconfiguração } \\
\hline - Necessidade de acompanhar a & - Abertura de filiais e escritórios & - Explorar novos conhecimentos; \\
expansão dos clientes para & comerciais; & $\begin{array}{l}\text { - Coordenação eficiente de uma base } \\
\text { global de ativos; } \\
\text { mercados internacionais; }\end{array}$ \\
- Aumento da concorrência. & $\begin{array}{l}\text { - Desenvolvimento de novos } \\
\text { processos e produtos; } \\
\text { - Transformação de rotinas; } \\
\text { exigências; } \\
\text { - Realinhamento dos ativos e recursos. }\end{array}$ \\
\hline
\end{tabular}

Figura 5. Articulação das CDs na internacionalização da empresa Beta

Fonte: Elaborado pelos autores

O processo de internacionalização das empresas analisadas, ainda que com as especificidades de cada uma, apresenta uma relevante vinculação com as abordagens comportamentais de inserção em mercados externos. Como postula o Modelo de Uppsala e de Networks, as empresas estudadas iniciaram esse processo a partir do desenvolvimento de relacionamentos com clientes e da aprendizagem experimental construída com base nessas atividades. Esses movimentos permitiram identificar e aproveitar oportunidades e aprimorar os relacionamentos com seus clientes. A orquestração de CDs, com destaque para elementos como, flexibilidade; articulação de conhecimentos; reconfiguração de ativos e modelos de negócios alinhados com as exigências externas, permitiram a essas empresas serem percebidas pelos seus clientes como parceiros estratégicos para acompanhá-los em uma expansão internacional. Essas capacidades também auxiliaram na organização interna para atender as exigências desses diferentes mercados.
Na Figura 6, expõe-se o framework, que relaciona as abordagens desenvolvidas neste estudo. Sendo assim, a trajetória organizacional das empresas e seus movimentos estratégicos realizados ao longo do tempo estão concatenados com o desenvolvimento e articulação de CDs. As CDs continuamente orquestradas pelas empresas habilitam a realização de movimentos estratégicos, as colocam em uma posição estratégica e de destaque perante seus clientes e, ao longo do tempo, permitem a expansão internacional e a permanência nestes mercados.

Por outro lado, esses movimentos estratégicos e os relacionamentos construídos influenciam no desenvolvimento de CDs e retroalimentam a manutenção e incremento dessas capacidades. Assim, há uma relação entre essas abordagens que possibilitam um bom desempenho, com a manutenção e crescimento da organização em âmbito doméstico e internacional.

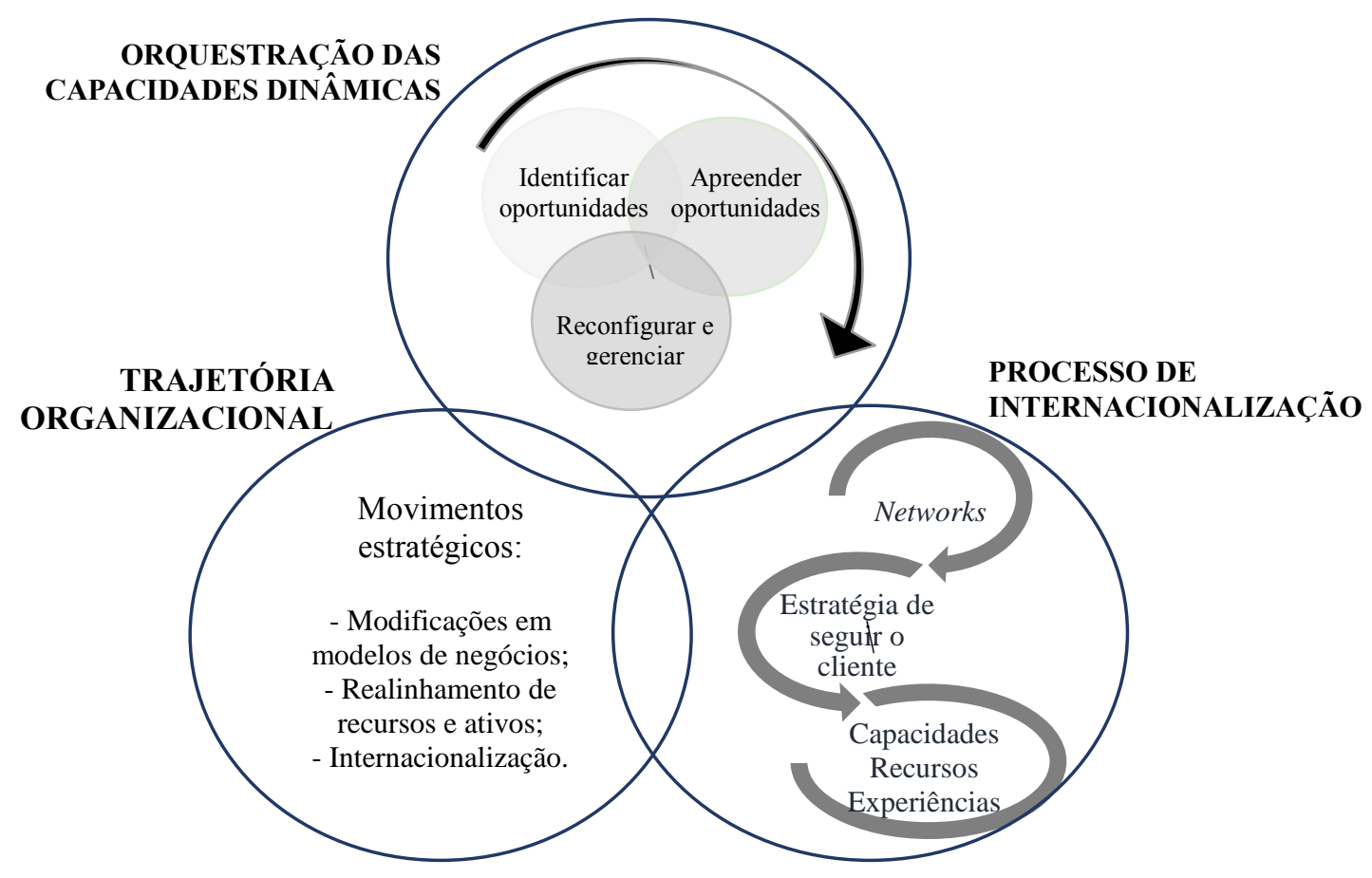

Figura 6. Framework da relação entre CDs, Trajetória Organizacional e Internacionalização Fonte: Elaborado pelos autores 


\section{CONSIDERAÇÕES FINAIS}

Este estudo teve o objetivo de compreender o papel das CDs no processo de internacionalização organizacional, como também quais os elementos e capacidades são desenvolvidos pelas empresas que se internacionalizam e se mantêm competitivas nos mercados externos. As pesquisas, predominantemente, avaliam a internacionalização por meio da inserção e o início do processo. No entanto, há uma necessidade de se avaliar a perenidade dessas atividades e suas influências para o crescimento integral da empresa. A presente pesquisa colaborou para essa análise, averiguando que, a articulação das CDs e as estratégias adequadas adotadas pelos casos estudados, permitiram às empresas se adaptarem aos mercados internacionais e orquestrarem seus ativos e recursos para abordarem as novas demandas. A associação entre internacionalização e a perspectiva das CDs se apresentou como um campo propício para estudo, visto que a orquestração dessas capacidades fortalece as empresas e auxilia na sustentação de atividades em mercados internacionais.

O estudo contribui para as literaturas de CDs e NIS nas indicações dos recursos organizacionais necessários para a inserção e atuação internacional e as maneiras de desenvolvimento e articulação das CDs no contexto doméstico e internacional. A compreensão da internacionalização dentro da ótica de recursos e capacidades colabora para a ampliação de análises que encadeiam as duas vertentes de pesquisa. $\mathrm{O}$ estudo evidenciou que as CDs desenvolvidas pelas organizações foram ampliadas após o ingresso em mercados internacionais e essas capacidades exerceram influência na ampliação dos NIs desenvolvidos. A internacionalização auxilia no incremento das CDs ao longo do tempo, assim como, a orquestração de CDs possibilita o ingresso, a permanência e expansão das atividades em mercados externos, refletindo na competitividade organizacional.

A pesquisa realizada destacou a premência da construção e do gerenciamento de CDs para o sucesso nas atividades internacionais das empresas. As CDs ligadas à exploração e articulação de conhecimentos; tomada de decisões estratégicas e à flexibilidade organizacional para modificar e realinhar rotinas, permitiram às empresas se incorporar localmente em contextos estrangeiros. Além disso, os recursos organizacionais colaboradores, sua formação e às estruturas tecnológicas alocadas se mostraram relevantes para auxiliar a ampliação das vantagens das empresas nos mercados internacionais.

Em termos de implicações para a investigação organizacional, no âmbito da gestão estratégica, o estudo apresentou evidências da articulação das CDs nas práticas das organizações. Tornou-se possível visualizar diversos meios e mecanismos pelos quais as CDs se manifestam e influenciam no desenvolvimento e crescimento das empresas para os mercados internacionais. Em relação às implicações gerenciais, pode-se perceber que, a construção das CDs, estratégias e as percepções de necessidade de mudanças ainda é realizada, em alguma medida, de forma inconsciente e não totalmente planejada nas empresas. 0 estudo contribuiu para demonstrar que, com o entendimento dos elementos das CDs, seu papel na competitividade e a forma com que a empresa se desenvolve ao longo do tempo, torna-se possível converter a construção e articulação dessas capacidades de forma mais deliberada nas empresas, tal como transformá-las em um processo mais cultural e enraizado na estrutura organizacional.

A despeito das contribuições apresentadas, há algumas limitações do estudo realizado. Estudos quantitativos, aliados a abordagem qualitativa, podem subsidiar outras formas de visualizar as CDs e sua relação com a internacionalização. Outras empresas de diferentes portes e/ou estruturas também podem trazer contribuições distintas para contrapor os achados a respeito da relação entre as abordagens. Complementarmente, os estudos longitudinais, proporcionariam, uma observação mais densa da construção e orquestração das CDs, trazendo uma gama de elementos para avaliar sua relação com a competitividade e a internacionalização, bem como ampliando a análise para a vinculação dessas capacidades com medidas de desempenho financeiro e desempenho internacional. Portanto, as lentes teóricas relacionadas nesta pesquisa podem auxiliar no desenvolvimento de novos estudos que contribuam para essas literaturas e nas práticas organizacionais. Espera-se ter contribuído para suscitar novas possibilidades de pesquisas e reflexões para os pesquisadores da área de gestão estratégica e permitir a evolução desse campo de pesquisa emergente e promissor. 


\section{REFERÊNCIAS}

Al-Aali, A., \& Teece, D. J. (2014). International entrepreneurship and the theory of the (long-lived) international firm: a capabilities perspective. Entrepreneurship Theory and Practice, 38(1), 95-116.

Andersen, O., \& Buvik, A. (2002). Firms' internationalization and alternative approaches to the international customer/market selection. International Business Review, 11(3), 347-363.

Augier, M., \& Teece, D. J. (2007). Dynamic capabilities and multinational enterprise: Penrosean insights and omissions. Management International Review, 47(2), 175-192.

Björkman, I., \& Forsgren, M. (2000). Nordic international business research: a review of its development. International Studies of Management \& Organization, 30(1), 6-25.

Blomstermo, A., Deo Sharma, D., \& Sallis, J. (2006). Choice of foreign market entry mode in service firms. International Marketing Review, 23(2), 211-229.

Boehe, D. M. (2016). The internationalization of service firms from emerging economies: An internalization perspective. Long Range Planning, 49(5), 559-569.

Butler, B., \& Soontiens, W. (2015). Offshoring of higher education services in strategic nets: A dynamic capabilities perspective. Journal of World Business, 50(3), 477-490.

Chakrabarty, S., \& Wang, L. (2012). The long-term sustenance of sustainability practices in MNCs: A dynamic capabilities perspective of the role of R\&D and internationalization. Journal of Business Ethics, 110(2), 205-217.

Chang, K. H., Chen, Y. R., \& Huang, H. F. (2015). Information technology and partnership dynamic capabilities in international subcontracting relationships. International Business Review, 24(2), 276286.

Coviello, N. E., \& Munro, H. J. (1995). Growing the entrepreneurial firm: networking for international market development. European journal of marketing, 29(7), 49-61.

Crick, D. (2009). The internationalisation of born global and international new venture SMEs. International Marketing Review, 26(4/5), 453476.
Da Costa, P. R.; Porto, G. S. (2014). Governança tecnológica e cooperabilidade nas multinacionais brasileiras. RAE-Revista de Administração de Empresas, 54(2).

Di Stefano, G., Peteraf, M., \& Verona, G. (2010). Dynamic capabilities deconstructed: a bibliographic investigation into the origins, development, and future directions of the research domain. Industrial and corporate change, 19(4), 1187-1204.

El Akremi, A., Perrigot, R., \& Piot-Lepetit, I. (2015). Examining the drivers for franchised chains performance through the lens of the dynamic capabilities approach. Journal of Small Business Management, 53(1), 145-165.

Eriksson, K., Johanson, J., Majkgard, A., \& Sharma, D. D. (1997). Experiential knowledge and cost in the internationalization process. Journal of international business studies, 337-360.

Eriksson, T., Nummela, N., \& Saarenketo, S. (2014). Dynamic capability in a small global factory. International Business Review, 23(1), 169-180.

Evers, N., Andersson, S., \& Hannibal, M. (2012). Stakeholders and marketing capabilities in international new ventures: evidence from Ireland, Sweden and Denmark. Journal of International Marketing, 20(4), 46-71.

Fan, D., Cui, L., Li, Y., \& Zhu, C. J. (2016). Localized learning by emerging multinational enterprises in developed host countries: A fuzzy-set analysis of Chinese foreign direct investment in Australia. International Business Review, 25(1), 187203.

Fang, E. E., \& Zou, S. (2009). Antecedents and consequences of marketing dynamic capabilities in international joint ventures. Journal of International Business Studies, 40(5), 742-761.

Floriani, D. E.; Borini, F. M.; Fleury, M. T. L. O processo de internacionalização como elemento gerador de capacidades dinâmicas: o caso da WEG na Argentina e na China. Revista Brasileira de Gestão de Negócios, 11(33).

Frasquet, M., Dawson, J., \& Mollá, A. (2013). Postentry internationalisation activity of retailers: An assessment of dynamic capabilities. Management Decision, 51(7), 1510-1527.

Griffith, D. A., \& Harvey, M. G. (2001). A resource perspective of global dynamic capabilities. Journal of International Business Studies, 32(3), 597-606. 
Grönroos, C. (1999). Internationalization strategies for services. Journal of services marketing, 13(4/5), 290297.

Helfat, C. E., Finkelstein, S., Mitchell, W., Peteraf, M., Singh, H., Teece, D., \& Winter, S. G. (2009). Dynamic capabilities: Understanding strategic change in organizations. John Wiley \& Sons.

Huijs, M., Jansen, S., \& Brinkkemper, S. (2015, June). Internationalization and Export of Software Products. In International Conference of Software Business (pp. 207-222). Springer, Cham.

Jantunen, A., Puumalainen, K., Saarenketo, S., \& Kyläheiko, K. (2005). Entrepreneurial orientation, dynamic capabilities and international performance. Journal of International Entrepreneurship, 3(3), 223-243.

Johanson, J., \& Mattsson, L. G. (1988). Internationalisation in industrial systems-a network approach. In Hood, N.; Vahlne, J.E. (Ed.). Strategies in global competition. London: Croom Helm, 287-314.

Johanson, J., \& Vahlne, J. E. (2009). The Uppsala internationalization process model revisited: From liability of foreignness to liability of outsidership. Journal of international business studies, 40(9), 1411-1431.

Khalid, S., \& Larimo, J. (2012). Firm specific advantage in developed markets dynamic capability perspective. Management International Review, 52(2), 233-250.

Khavul, S., Peterson, M., Mullens, D., \& Rasheed, A. A. (2010). Going global with innovations from emerging economies: investment in customer support capabilities pays off. Journal of International Marketing, 18(4), 22-42.

Kim, J. U., \& Aguilera, R. V. (2016). Foreign location choice: Review and extensions. International Journal of Management Reviews, 18(2), 133-159.

Knebel, P. (2010). Dos grãos aos chips: a história da tecnologia e da inovação no Rio Grande do Sul. Porto Alegre: EdiPUCRS.

Knight, G. A. (2001). Entrepreneurship and strategy in the international SME. Journal of international management, 7(3), 155-171.

Knudsen, T., \& Madsen, T. K. (2002). Export strategy: a dynamic capabilities perspective. Scandinavian Journal of Management, 18(4), 475-502.

Lessard, D., Teece, D. J., \& Leih, S. (2016a). The Dynamic Capabilities of Meta-Multinationals. Global Strategy Journal, 6(3), 211-224.
Lessard, D., Teece, D. J., \& Leih, S. (2016b). Introduction to special topic forum on developing the dynamic capabilities of global companies across levels and locations. Global Strategy Journal, 6(3), 165-167.

Lew, Y. K., Sinkovics, R. R., \& Kuivalainen, O. (2013). Upstream internationalization process: roles of social capital in creating exploratory capability and market performance. International Business Review, 22(6), 1101-1120.

Lommelen, T., \& Matthyssens, P. (2005). The internationalization process of service providers: a literature review. In Research on international service marketing: a state of the art (pp. 95-117). Emerald Group Publishing Limited.

Luo, Y. (2001). Dynamic capabilities in international expansion. Journal of World Business, 35(4), 355-378.

Majkgård, A., \& Sharma, D. D. (1998). Clientfollowing and market-seeking strategies in the internationalization of service firms. Journal of Businessto-Business Marketing, 4(3), 1-41.

Monferrer, D., Blesa, A., \& Ripollés, M. (2015). Born globals trough knowledge-based dynamic capabilities and network market orientation. $B R Q$ Business Research Quarterly, 18(1), 18-36.

Moreira, M. Z., \& Forte, S. H. A. C. (2006). Competitividade internacional baseada em recursos: estudo da relação entre os recursos e as estratégias de internacionalização nas maiores empresas exportadoras do setor calçadista brasileiro. Internext Revista Eletrônica de Negócios Internacionais, 1(1), 77102.

Parente, R. C., Baack, D. W., \& Hahn, E. D. (2011). The effect of supply chain integration, modular production, and cultural distance on new product development: A dynamic capabilities approach. Journal of International Management, 17(4), 278-290.

Pehrsson, T., Ghannad, N., Pehrsson, A., Abt, T., Chen, S., Erath, F., \& Hammarstig, T. (2015). Dynamic capabilities and performance in foreign markets: Developments within international new ventures. Journal of International Entrepreneurship, 13(1), 28-48.

Peteraf, M., Di Stefano, G., \& Verona, G. (2013). The elephant in the room of dynamic capabilities: Bringing two diverging conversations together. Strategic management journal, 34(12), 1389-1410.

Pinho, J. C., \& Prange, C. (2016). The effect of social networks and dynamic internationalization capabilities 
O Papel das Capacidades Dinâmicas na Internacionalização de Empresas

on international performance. Journal of World Business, 51(3), 391-403.

Pitelis, C. N., \& Teece, D. J. (2010). Cross-border market co-creation, dynamic capabilities and the entrepreneurial theory of the multinational enterprise. Industrial and corporate change, 19(4), 1247-1270.

Pla-Barber, J., \& Escribá-Esteve, A. (2006). Accelerated internationalisation: evidence from a late investor country. International Marketing Review, 23(3), 255-278.

Prange, C., \& Verdier, S. (2011). Dynamic capabilities, internationalization processes and performance. Journal of World Business, 46(1), 126133.

Rodriguez, C. M.; A. Wise, J.; Martinez, C. R. (2013). Strategic capabilities in exporting: an examination of the performance of Mexican firms. Management Decision, 51(8), 1643-1663.

Swoboda, B., \& Olejnik, E. (2016). Linking processes and dynamic capabilities of international SMEs: the mediating effect of international entrepreneurial orientation. Journal of Small Business Management, 54(1), 139-161.

Tallott, M., \& Hilliard, R. (2016). Developing dynamic capabilities for learning and internationalization: A case study of diversification in an SME. Baltic Journal of Management, 11(3), 328-347.

Teece, D. J. (2007). Explicating dynamic capabilities: the nature and microfoundations of (sustainable) enterprise performance. Strategic management journal, 28(13), 1319-1350.

Teece, D. J. (2014a). A dynamic capabilities-based entrepreneurial theory of the multinational enterprise. Journal of International Business Studies, 45(1), 8-37.

Teece, D. J. (2014b). The foundations of enterprise performance: Dynamic and ordinary capabilities in an (economic) theory of firms. The Academy of Management Perspectives, 28(4), 328-352.

Teece, D. J.; Pisano, G.; Shuen, A. (1997). Dynamic capabilities and strategic management. Strategic management journal, 18(7), 509-533.

Thanos, I. C., Dimitratos, P., \& Sapouna, P. (2017). The implications of international entrepreneurial orientation, politicization, and hostility upon SME international performance. International small business journal, 35(4), 495-514.
Turcan, R. V., \& Juho, A. (2016). Have we made it? Investigating value-creating strategies in early internationalizing ventures. Competitiveness Review, 26(5), 517-536.

Vahlne, J. E., \& Ivarsson, I. (2014). The globalization of Swedish MNEs: Empirical evidence and theoretical explanations. Journal of International Business Studies, 45(3), 227-247.

Vahlne, J. E., \& Johanson, J. (2017). From internationalization to evolution: The Uppsala model at 40 years. Journal of International Business Studies, 48(9), 1087-1102.

Vahlne, J. E., \& Jonsson, A. (2017). Ambidexterity as a dynamic capability in the globalization of the multinational business enterprise (MBE): Case studies of AB Volvo and IKEA. International Business Review, 26(1), 57-70.

Villar, C., Alegre, J., \& Pla-Barber, J. (2014). Exploring the role of knowledge management practices on exports: A dynamic capabilities view. International Business Review, 23(1), 38-44.

Weerawardena, J., Mort, G. S., Liesch, P. W., \& Knight, G. (2007). Conceptualizing accelerated internationalization in the born global firm: A dynamic capabilities perspective. Journal of world business, 42(3), 294-306.

Weerawardena, J., Mort, G. S., Salunke, S., Knight, G., \& Liesch, P. W. (2015). The role of the market sub-system and the socio-technical sub-system in innovation and firm performance: a dynamic capabilities approach. Journal of the Academy of Marketing Science, 43(2), 221-239.

Welch, C., \& Paavilainen-Mäntymäki, E. (2014). Putting process (back) in: research on the internationalization process of the firm. International Journal of Management Reviews, 16(1), 2-23.

Welch, D. E., \& Welch, L. S. (1996). The internationalization process and networks: a strategic management perspective.Journal of international marketing, 11-28.

Werlang, N. B. W., Dalbosco, I. B., Floriani, D. E., \& Rossetto, C. R. (2014). Capacidades dinâmicas e o processo de internacionalização inward de uma multinacional argentina para o Brasil. Internext, 9(1), 22-39.

Zimmermann, A., \& Birkinshaw, J. (2016). Reconciling Capabilities and Ambidexterity Theories: A Multi-level Perspective. In Teece, D. J. \& Leih, S. (Org.). The Oxford Handbook of Dynamic Capabilities. Oxford Hanbook Online. 


\section{SOBRE OS AUTORES}

- Caroline Kretschmer - University of Vale do Rio dos Sinos - UNSINOS, Rio Grande do Sul, (Brazil). E-mail: carol.kretschmer@brturbo.com.br Orcid id: http://orcid.org/0000-0002-3318-0947.

- Ivan Lapuente Garrido - University of Vale do Rio dos Sinos - UNISINOS, Rio Grande do Sul, (Brazil). E-mail: igarrido@unisinos.br Orcid id: http://orcid.org/0000-0003-3741-7961

\section{The Role Of Dynamic Capabilities In Business Internationalization}

Caroline Kretschmer e Ivan Lapuente Garrido

University of the Valley of the Sinos River - Unisinos, Rio Grande do Sul, (Brazil)

\section{ARTICLE DETAILS}

\section{Invited paper:}

Received: 23 August 2018

Accepted: 11 February 2019

Available online March: 26th 2019

\section{Scientifc Editor}

Ilan Avrichir

\section{Keywords:}

Dynamic Capabilities

Internationalization

Strategy

\begin{abstract}
The objective of this research is to contribute to the Dynamic Capabilities and International Business' literature. This article presents the organizational resources that allow insertion and permanence in external markets, as well as the capabilities developed by companies that are internationalized and remained in the international context. Therefore, was articulated the theoretical lens of Dynamic Capabilities and their relationship with internationalization, besides to combine the literature about organizational internationalization. Empirical research was developed through the analysis of the internationalization process and the strategic behavior of two Brazilian companies. A multiple and qualitative case study was carried out, data were collected through semi-structured interviews, documents, and analyzed through content analysis. As a result, the internationalization proved viable and based on the Dynamic Capabilities' articulation that shapes a competitive organizational architecture and in the constant managers' perception about new opportunities of international action coming from their clients. The article contributes to the two theoretical aspects by proposing a framework to concatenate them, evidencing the resources, paths, and capabilities necessary for the sustainability and expansion in international contexts, as well as the relevance of the Dynamic Capabilities in the business internationalization.
\end{abstract}

Kretschmer, C., \& Garrido, I. (2019). O Papel das Capacidades Dinâmicas na Internacionalização de Empresas. Revista Eletrônica de Negócios Internacionais, 14(2), 111-127. doi:http://dx.doi.org/10.18568/internext.v14i2.468 


\section{PÊNDICE - Roteiro de entrevistas}

\section{Identificar como se desenvolveu o processo e as estratégias de internacionalização das empresas estudadas.}

- Solicitar ao entrevistado que conte como ocorreu a internacionalização da empresa e como se desenvolve as atividades interacionais;

- Questionar quais foram os motivos para a internacionalização da empresa, bem como o impacto, vantagens e dificuldades percebidas:

Questionar quais adaptações foram necessárias para a internacionalização;

Questionar a respeito dos modos de entrada utilizados;

Questionar a respeito dos critérios para seleção dos mercados-alvo;

Questionar quais as estratégias de marketing foram necessárias para atender os mercados externos;

Questionar a respeito dos aspectos macroambientais do mercado internacional (culturais, políticos, sociais, macroecômicos);

Questionar a respeito dos concorrentes e do perfil dos clientes no mercado internacional;

Questionar a respeito do impacto das estratégias de internacionalização para o desempenho da empresa.

\section{Verificar a relação das CDs com os movimentos estratégicos apontados.}

Para cada movimento estratégico questionar:

- Questionar o que motivou esse movimento, de onde partiu a iniciativa;

Questionar se foram realizadas reuniões com clientes, fornecedores e terceiros vinculados a essa atividade;

- Questionar se houve algum tipo de mapeamento das necessidades de clientes e mercados, como foi efetuado reconhecimento das mudanças percebidas;

- Questionar como o conhecimento gerado nessa atividade e em outros movimentos efetuados pela empresa é gerado e disseminado entre os funcionários e nas unidades dispersas globalmente:

Questionar como a empresa estava estruturada para esse fato, divisão de tarefas e responsabilidades;

- Questionar a respeito de investimentos em P\&D e se há uma base externa de pesquisa que contemple clientes, fornecedores e complementares.

Questionar como a empresa se organizou/mobilizou para esse movimento, quais mudanças foram necessárias;

- Questionar como se deu o processo de gestão e as atividades funcionais;

Questionar a necessidade de desenvolver novos produtos, serviços e processos;

Questionar a necessidade de desenvolver e/ou adquirir novas tecnologias;

Questionar se houve alterações no modelo de negócio da empresa e se houve flexibilidade para tais alterações;

- Questionar sobre a tomada de decisões estratégicas;

- Questionar sobre alterações nas rotinas, como foram assimilados e disseminados os novos conhecimentos requeridos.

Questionar se, após a implementação desse movumento, fez-se necessário algum ajuste no que tange:

* desenvolver processos de codificação e disseminação de conhecimento para alterar conjuntos de rotinas;

* explorar os novos conhecimentos desenvolvidos e os resultados alcançados por meio da sua articulação

* desenvolver e manter cadeias de valor (questionar como se estabeleceu)

* estabelecer networks com parceiros externos (questionar como ocorre/ocorreu esses relacionamentos)

* como foram tomadas as decisões (centralização) e flexibilidade para as mudanças;

* como se deu o gerenciamento da propriedade intelectual e da transferência de tecnologias.

- Questionar se a empresa já abandonou alguma combinação de recursos e rotinas e os motivos que conduziram a essa mudança. Fonte: Elaborado pelos autores 